\title{
Defining Well Clear Separation for Unmanned Aircraft Systems Operating with Non-cooperative Aircraft
}

\author{
Christine C. Chen ${ }^{1}$, Bilal Gill ${ }^{2}$, and Matthew W. M. Edwards ${ }^{3}$ \\ Lincoln Laboratory, Massachusetts Institute of Technology, Lexington, MA 02420, USA \\ Samantha Smearcheck ${ }^{4}$, Tony Adami ${ }^{5}$, and Sean Calhoun ${ }^{6}$ \\ CAL Analytics, Beavercreek, OH, 45431, USA \\ M. Gilbert $\mathrm{Wu}^{7}$ and Andrew C. Cone ${ }^{8}$ \\ NASA Ames Research Center, Moffett Field, CA 94035, USA \\ Seungman Lee $^{9}$ \\ Crown Consulting Inc., NASA Ames Research Center, Moffett Field, CA 94035, USA
}

\begin{abstract}
Detect-and-Avoid (DAA) systems are essential to the safe operations of Unmanned Aircraft Systems, and have the objectives of mitigating collisions with and remaining Well Clear of manned aircraft. This paper analyzes four candidate DAA Well Clear definitions for noncooperative aircraft using mitigated performance metrics of DAA systems. These DAA Well Clear definitions were proposed in previous work based on their unmitigated collision risk and maneuver initiation range. In this work they are evaluated using safety and operational suitability metrics computed from a large number of representative encounters. Results suggest that although the four candidate DAA Well Clear definitions provide comparable safety, the alerting characteristics give preference for the DAA Well Clear definition without a temporal parameter.
\end{abstract}

\section{Introduction}

Detect and Avoid (DAA) systems are essential for safe integration of UAS into the National Airspace System (NAS). DAA Systems provide surveillance, alerts, and guidance to Unmanned Aircraft Systems (UAS) to help them remain Well Clear of other aircraft. DAA Well Clear (DWC) is a separation standard used in DAA systems that is designed as an alternative means of compliance with see-and-avoid regulations to support safe operations and avoid collisions between unmanned aircraft and manned aircraft. Previously, a process based on safety performance and operational suitability [1] [2] was followed to select and fine-tune DWC standards for UAS operations in non-terminal

\footnotetext{
${ }^{1}$ Associate Technical Staff, Surveillance Systems

${ }^{2}$ Subcontractor, Surveillance Systems, AIAA Member

${ }^{3}$ Technical Staff, Surveillance Systems, Senior AIAA Member

${ }^{4}$ System Analyst

${ }^{5}$ System Analyst

${ }^{6}$ Director

${ }^{7}$ Research Engineer, Aviation Systems, AIAA Member

${ }^{8}$ Research Engineer, Aviation Systems, AIAA Member

${ }^{9}$ Senior Research Scientist, Senior AIAA Member
}

DISTRIBUTION STATEMENT A. Approved for public release. Distribution is unlimited.

This material is based upon work supported by the National Aeronautics and Space Administration under Air Force Contract No. FA8702-15-D-0001. Any opinions, findings, conclusions or recommendations expressed in this material are those of the author(s) and do not necessarily reflect the views of the National Aeronautics and Space Administration. 
areas. These standards are documented in the Phase 1 Minimum Operational Performance Standards (MOPS) for DAA systems [3] and air-to-air radar [4] published by the RTCA Special Committee 228 (SC-228) in 2017, as well as the corresponding Technical Standard Orders (TSO), TSO-C211 and TSO-C212 published by the Federal Aviation Administration (FAA) in October 2017. The UAS in the Phase 1 MOPS are assumed to be equipped with Automatic Dependent Surveillance-Broadcast (ADS-B) In, airborne active surveillance, and a high-power air-to-air radar that can detect aircraft without transponders (non-cooperative aircraft). Traffic Alert and Collision Avoidance System (TCAS) II Resolution Advisories (RAs) are optional. The Phase 1 UAS can operate atairspeeds up to 200 kts. Phase 2 work for extending the MOPS to additional UAS categories and operations is underway.

One of the main objectives of the Phase 2 development is to define an alternative DAA Well Clear (DWC) definition for UAS encountering non-cooperative aircraft. The DWC in the Phase 1 work was selected with considerations of interoperability with TCAS-II. To avoid triggering TCAS resolution advisories during an encounter (leading to DAA maneuvers), the DWC was defined to encompass a vast majority of the TCAS alerting volume. The resulting DWC is deemed very safe but may be unnecessarily large for encounters of UAS with non-cooperative aircraft, because TCAS-II cannot detect non-cooperative aircraft and hence, noncooperative aircraft will not trigger TCAS RAs. Four DWC candidates were proposed for additional evaluation based on a previous study performed by NASA, Lincoln Laboratory, and Adaptive Aerospace [5]. These four candidate DWC were proposed based on considerations of the unmitigated collision rate, maneuver initiation range, and other factors.

Another objective of the Phase 2 DAA MOPS is to define requirements for operations of UAS equipped with low cost, size, weight, and performance (C-SWaP) sensors, or low C-SWaP UAS. Many UAS are too small or budgetconstrained to carry the large, high-power radar required by the Phase 1 MOPS. Examples are the Aerosonde and Shadow UAS. Low C-SWaP sensors tend to have shorter detection. However, the limited detection range of low CSWaP sensors may be acceptable when considering the reduced closing speed of encounters given the reduced low C-SWaP UAS speeds. Reconsidering the DAA Well Clear definition in the context of non-cooperative aircraft may help preserve the alerting timeline in the presence of limited surveillance range.

The DWC definition considered in this work is for UAS against non-cooperative aircraft. It is anticipated to be applicable to both Phase 1 UAS and low C-SWaP UAS. A reduced DWC can potentially reduce the required radar surveillance volume for Phase 1 UAS. However, a potential downside of a reduced DWC is a reduction in the DAA systems' overall safety.

This paper presents an evaluation of four candidate DAA Well Clear (DWC) definitions [5] using encounters representative of both a low-C-SWaP and a Phase 1 UAS encountering a non-cooperative aircraft. The focus of this analysis is to assess the safety and operational suitability metrics achieved using these candidate DWC definitions. These metrics provide an indication of whether the system will be able to remain Well Clear of other aircraft without alerting or maneuvering unnecessarily, which could interfere with the operations of the UAS, other aircraft, or air traffic control (ATC). Section II reviews the approach used to evaluate the DWC candidates, including the encounter sets and pilot response model that was used. Results are presented in Section III, and conclusions and future work are discussed in Section IV.

\section{Approach}

The DWC candidates are shown in Table 1. DWC1 and DWC2 are the two primary candidates, both achieving a desirable unmitigated collision risk of 5\%. Unmitigated collision risk is the likelihood that two aircraft would violate the DAA Well Clear definition if neither aircraft used a DAA system. DWC1 achieves the minimum maneuver initial range (MIR), which is the range between aircraft when the UAS must start maneuvering away in order to maintain DWC, whereas DWC2 is simple because it does not have a time component. DWC3 and DWC4 are backup candidates carried forward from previous analysis in case DWC1 and DWC2 do not perform well in certain categories of metrics. DWC3 achieves a "risky" unmitigated collision risk of 7\%. It was once proposed for terminal area UAS operations. DWC4 achieves an unmitigated collision risk between 3.5-4\% and was considered a "safer" candidate that achieves an unmitigated collision risk of less than 5\%. In addition to the four DWC candidates selected in [5], the Phase 1 DWC definition was also evaluated for comparison.

Table 1 DWC Candidates

\begin{tabular}{|l|c|c|c|c|c|}
\hline & DWC1 & DWC2 & DWC3 & DWC4 & Phase 1 \\
\hline HMD $^{*}$ & $2000 \mathrm{ft}$ & $2200 \mathrm{ft}$ & $1500 \mathrm{ft}$ & $2500 \mathrm{ft}$ & $4000 \mathrm{ft}$ \\
\hline$h^{*}$ & $450 \mathrm{ft}$ & $450 \mathrm{ft}$ & $450 \mathrm{ft}$ & $450 \mathrm{ft}$ & $450 \mathrm{ft}$ \\
\hline$\tau_{\text {mod }} *$ & $15 \mathrm{~s}$ & $0 \mathrm{~s}$ & $15 \mathrm{~s}$ & $25 \mathrm{~s}$ & $35 \mathrm{~s}$ \\
\hline
\end{tabular}


The DWC candidates are defined by thresholds of three parameters: horizontal miss distance (HMD), vertical separation threshold $(h)$, and $\tau_{m o d}$. HMD*, h*, and $\tau_{m o d} *$ are the specific values of these parameters used to define each DWC candidate. The HMD parameter is the predicted minimum horizontal distance during an encounter, assuming constant velocities and straight line flight. The $h$ parameter is the current altitude difference between the two aircraft. For all candidates, $h *$ is $450 \mathrm{ft}$, which is equal to the component used in the Phase 1 MOPS. The definition of $\tau_{\text {mod }}$ is

$$
\tau_{\text {mod }}=\left\{\begin{array}{c}
-\frac{r^{2}-D_{\text {mod }}^{2}}{r \dot{r}}, r>D_{\text {mod }} \\
0, r \leq D_{\text {mod }}
\end{array}\right.
$$

where $r$ and $\dot{r}$ are horizontal range and range rate, respectively, between the UAS and the intruder. $D_{\text {mod }}$ is the distance modification and defines the radius of a cylinder around the UAS. In this analysis, $D_{\text {mod }}$ is set equal to the horizontal miss distance threshold, HMD *, in the DWC definitions. A loss of DWC (LoDWC) occurs when all three parameters, computed from aircraft states, fall below their respective thresholds.

One million uncorrelated encounters between one low-C-SWaP UAS and one non-cooperative intruder, and one million uncorrelated encounters between one Phase 1 UAS and one non-cooperative intruder were simulated. Uncorrelated encounters are situations where intervention from ATC is unlikely, such that aircraft blunder into close proximity. The UAS trajectory is sampled from NASA's Airspace Concept Evaluation System (ACES) UAS database [6], and the intruder trajectory is sampled from Lincoln Laboratory's Uncorrelated Encounter Model [7].

Next, the encounters are simulated using the Detect and AvoID Alerting Logic for Unmanned Systems (DAIDALUS) as the DAA alerting and guidance algorithm [8]. DAIDALUS is a reference algorithm used to validate the Phase 1 MOPS, and was used in this study as a representative DAA algorithm. DAIDALUS generates maneuver guidance by projecting candidate vertical and horizontal DAA maneuvers to determine which would result in conflicts, and which can be used to aid the pilot in resolving the situation manually. The evaluation for each DWC consisted of two scenarios: one nominal (or unmitigated) and one mitigated.

DAIDALUS issues three types of alerts in increasing levels of severity: preventive, corrective, and warning. The lowest level, preventive, is primarily used to alert the pilot to not maneuver vertically when the aircraft are separated vertically by $450-700$ feet. The second level, corrective, indicates that a LoDWC is predicted and an avoidance maneuver is necessary, but there is still time for coordination with ATC. The highest level, warning, indicates that a LoDWC is imminent, an immediate avoidance maneuver is needed, and coordination with ATC before maneuvering is not a requirement. Upon alerting, DAIDALUS generates corresponding preventive, corrective, and warning guidance indicating a range of conflict-free headings and altitudes for a pilot to select from in order to maintain DWC separation. In the event that a LoDWC cannot be avoided, DAIDALUS also generates regain DWC guidance, a range of heading or altitude that can be executed to increase separation at CPA and regain DWC effectively.

DAIDALUS alerts are issued based on a buffered DWC volume. Specifically, the HMD* used by the DAA alerting and guidance algorithm is scaled by a factor of 1.52 to be consistent with the parameters referenced in the Phase 1 MOPS [3]. This buffer is meant to guard against maneuvering intruders and surveillance uncertainties (there are none in this work). In the simulation, the ownship only maneuvers when a corrective or warning alert is received. For maneuver guidance computation, the low C-SWaP UAS turn rate was assumed to be $7 \mathrm{deg} / \mathrm{sec}$, which is suitable for UAS speeds from 40 to $100 \mathrm{kts}$ and results in approximately the same bank angle and load factor as the Phase 1 UAS turn rate, which was assumed to be $3 \mathrm{deg} / \mathrm{sec}$.

The simulations of low C-SWaP UAS were performed using perfect (truth) surveillance, i.e., with no track uncertainty or range limitations, in order to evaluate the mitigated performance of low C-SWaP without confounding factors (such as limited surveillance range or sensor noise). However, to assess the potential impacts of limited detection ranges on safety, simulations were also run with $2 \mathrm{NM}, 3 \mathrm{NM}$, and $4 \mathrm{NM}$ surveillance ranges applied; the results of these simulations are presented in Section III. The simulations of Phase 1 UAS were run with truth surveillance (i.e., no track uncertainty) constrained by a Phase 1 radar field, defined as $\pm 8 \mathrm{NM}$ range, $\pm 15^{\circ}$ elevation, and $\pm 110^{\circ}$ azimuth. The reason for using the Phase 1 radar surveillance volume is to faciliate comparison to a previous study of the Phase 1 UAS [9]. Upon completion of the simulations, metrics are computed to compare the performance of the DWC definitions. 


\section{A. Metrics}

The safety and operational suitability metrics provide an indication of whether the system will be able to operate safely without interfering with the operations of other aircraft and without causing DAIDALUS to alert unnecessarily. These metrics and their formulation are shown in Table 2 and

Table 3.

Table 2 Safety Metrics

\begin{tabular}{|c|c|}
\hline Metric & Notes \\
\hline \multirow[t]{3}{*}{ NMAC Risk Ratio } & $P($ NMAC|encounter, with mitigation $)$ \\
\hline & $\overline{P(N M A C \mid \text { encounter }, \text { without mitigation })}$ \\
\hline & $\begin{array}{l}\text { A Near Mid-Air Collision (NMAC) occurs when the separation between two aircraft } \\
\text { is less than } 500 \mathrm{ft} \text { horizontally and } 100 \mathrm{ft} \text { vertically [10]. If the ratio is less than one, } \\
\text { then the mitigated system reduces the risk of NMAC. For example, a risk ratio of } 0.1 \\
\text { indicates a } 90 \% \text { reduction in risk. Small values are desirable. }\end{array}$ \\
\hline \multirow[t]{3}{*}{ LoDWC Ratio } & $P($ LoDWC $\mid$ encounter, with mitigation $)$ \\
\hline & $\overline{P(\text { LoDWC } \mid \text { encounter }, \text { without mitigation })}$ \\
\hline & $\begin{array}{l}\text { Similar to the NMAC risk ratio, if the LoDWC risk ratio is less than one, then the } \\
\text { mitigated system reduces the risk of LoDWC. Small values are desirable. }\end{array}$ \\
\hline
\end{tabular}

Table 3 Operational Suitability Metrics

\begin{tabular}{|l|l|}
\hline Metric & Notes \\
\hline Alert Ratio & \multicolumn{1}{c|}{$\begin{array}{l}P(\text { Alert|encounter, with mitigation }) \\
P(\text { NMAC|encounter, without mitigation })\end{array}$} \\
$\begin{array}{l}\text { Given the same risk ratio, systems with lower alert ratios are desirable, since fewer } \\
\text { alerts indicate fewer unnecessary maneuvers. }\end{array}$ \\
\hline $\begin{array}{l}\text { Alerting time and range } \\
\text { relative to the LoDWC } \\
\text { point }\end{array}$ & $\begin{array}{l}\text { This metric can help inform alerting timeline requirements, and thus, sensor range } \\
\text { requirements. }\end{array}$ \\
\hline
\end{tabular}

\section{B. Encounters}

This study assessed two sets of encounters: one set with encounters between one low C-SWaP UAS and one noncooperative intruder, and the other set with encounters between one Phase 1 UAS and one non-cooperative intruder. All encounters contains $1 \mathrm{~Hz}$ aircraft states, and a majority of encounters last 180 seconds. These encounters were generated by pairing one projected UAS trajectory generated by NASA's UAS mission flights, and one intruder trajectory sampled from MIT Lincoln Laboratory's Uncorrelated Encounter Model [7]. NASA's UAS mission flights consist of 19 different types of missions, including aerial imaging and mapping, law enforcement, and air quality monitoring. The demand and mission profiles were generated based on subject matter experts' opinions and socioeconomic analysis [11]. The trajectories cover the entire continental US. These aircraft models are defined in a way similar to those in the Eurocontrol Base of Aircraft Data (BADA) [12]. In the low C-SWaP encounter set, two types of UAS were considered: the RA-7 AAI Shadow B and the MQ-19 AAI Aerosonde. The Phase 1 encounter set includes trajectories for seven types of UAS: Cessna 208 Caravan, Cessna 510 Citation Mustang, AAI Aerosonde, MQ-9A Reaper, RQ-4A Global Hawk, Shadow B, and Socata Trinidad.

The Uncorrelated Encounter Model is derived from radar data of observed aircraft operations under visual flight rules (VFR) in the National Airspace System. The model is sampled to produce random aircraft trajectories that are statistically representative of non-cooperative trajectories. Each encounter is specified by the initial positions and orientations of the two aircraft in the simulation and the nominal dynamic maneuvers that may occur leading up to the time of closest approach (TCA).

Filters were applied to the ownship and intruder speeds and altitudes to ensure that the dynamics of the sampled trajectories are within the bounds for low C-SWaP UAS or Phase 1 UAS, and the intruders they are expected to encounter. The intruder characteristics from the Uncorrelated Encounter Model are the same in both sets of encounters. The low C-SWaP UAS speeds are constrained to be between 40 and $100 \mathrm{kts}$, and the Phase 1 UAS speeds are 
constrained to be between 40 and $250 \mathrm{kts}, 50 \mathrm{kts}$ more than permitted by the Phase 1 MOPS so as to explore potential safety issues for faster UASs. The intruder speeds range from 0 to $170 \mathrm{kts}$, the 95 percentile speed for non-cooperative intruders in the Uncorrelated Encounter Model [7]. Intruders with zero speed represent aircraft like helicopters that are hovering. The encounters occur at altitudes between $500 \mathrm{ft}$ above ground level (AGL) and 10,999 ft mean sea level (MSL), in airspace classes $\mathrm{E}$ and G. Although Class $\mathrm{E}$ only goes up to $10,000 \mathrm{ft}$ when it is adjacent to class B or class C airspace, altitudes up to 10,999 feet were included to represent a few UAS missions that are flown slightly above $10,000 \mathrm{ft}$. The resulting altitude and speed distributions are shown in Ownship Type
Fig. 1 and
a) Altitudes
b) Speeds

c) Ownship Type

Fig. 2.

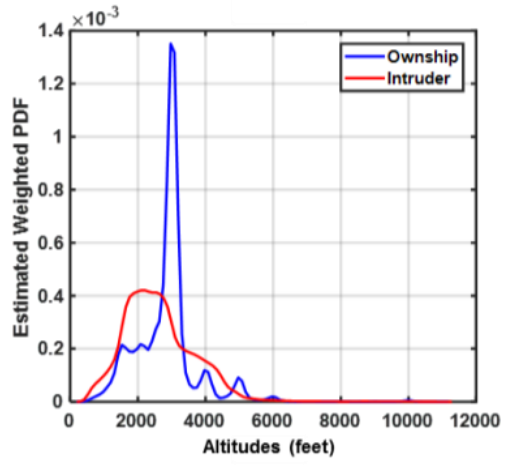

a) Altitudes

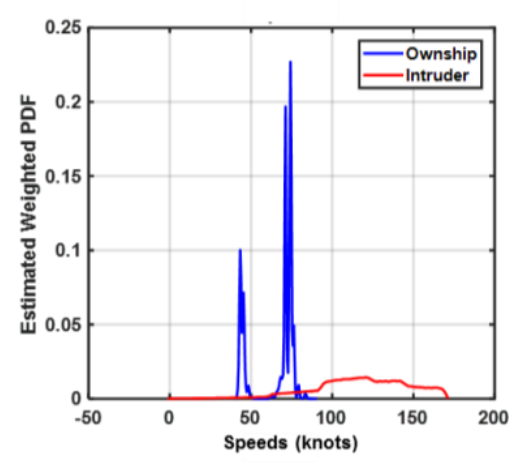

b) Speeds

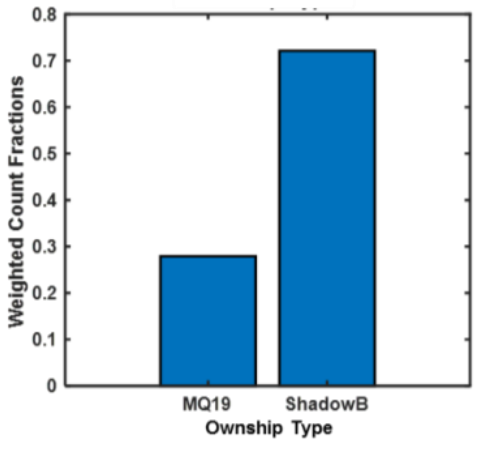

c) Ownship Type

Fig. 1 Low C-SWaP UAS Encounter Characteristics

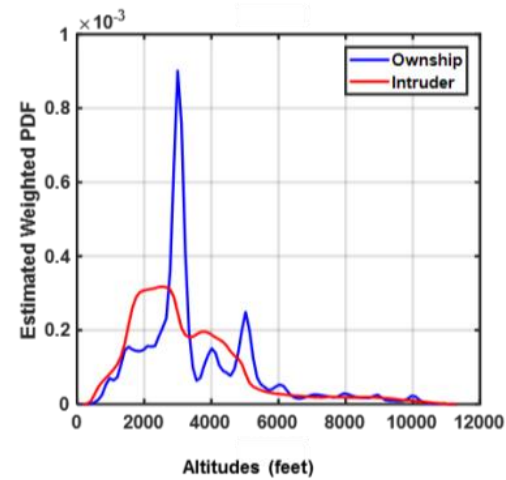

a) Altitudes

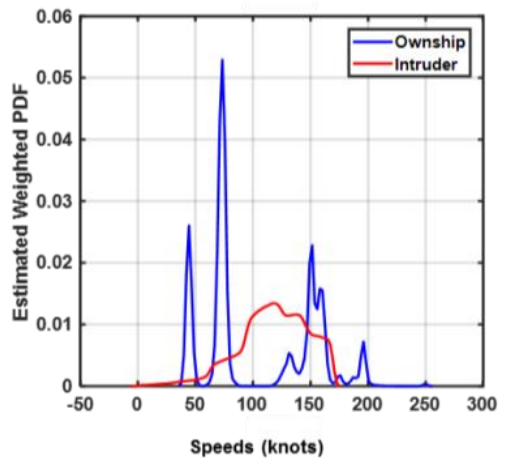

b) Speeds



c) Ownship Type

Fig. 2 Phase 1 UAS Encounter Characteristics

\section{Pilot Response Model}

Upon alerting, DAIDALUS provides guidance indicating a range of conflict-free headings and altitudes. The SC228 standard pilot model created by Lincoln Laboratory [13] is then used to select and execute an appropriate maneuver. Encounters with an alert within the first 5 seconds of the encounter are excluded from the analysis to ensure that the pilot response model is given adequate time to resolve the conflict. Only horizontal maneuvers were executed because vertical maneuvers against non-cooperative intruders are much less robust in most situations due to the uncertainties in non-cooperative sensors' vertical measurements. The pilot response model was executed in deterministic mode, meaning that the ownship always maneuvers horizontally in the direction of the minimum suggested maneuver. In the event that the minimum suggestion is inconclusive, the ownship will turn left, as a preference for left turns was observed in human-in-the-loop experiments [13]. For this analysis, the pilot model chooses the minimum heading change suggested by the guidance bands. Although variability was observed in humanin-the loop experiments, the minimum heading change was used in the analysis to isolate the effect of the DAA Well Clear definition from other parameters.

After the first alert is received, the pilot response model has a $5 \mathrm{sec}$ initial delay representing the time it takes the pilot to perceive the alert and devise a plan. For corrective alerts, there is an $11 \mathrm{sec}$ ATC coordination time representing the time it takes the pilot to communicate the intended maneuver with ATC and receive approval. The ATC coordination time is then followed by a $3 \mathrm{sec}$ execution delay representing the time it takes the pilot to enter the 
maneuver command into the control station and transmit this command to the UAS. The ownship may perform multiple maneuvers per encounter to resolve a conflict. The time between pilot response model decisions is determined by the alert state, as shown in

Table 4. For example, if the pilot model chooses a maneuver during a warning alert state, then the situation will be reevaluated after 9 seconds (the decision update period), and a different subsequent maneuver can be issued at that time, if needed. All delays and times (e.g., the $11 \mathrm{sec}$ ATC coordination time, $3 \mathrm{sec}$ execution delay, etc.) are the mean values of distributions observed from human-in-the loop studies used to build the pilot response model [13].

Table 4 Pilot Response Model Decision Update Times

\begin{tabular}{|l|c|}
\hline \multicolumn{1}{|c|}{ Alert Condition } & Decision Update Period (s) \\
\hline No Alert & 24 \\
\hline Preventive Alert & 15 \\
\hline Corrective Alert & 9 \\
\hline Warning Alert & 9 \\
\hline Regain DAA Well Clear Guidance & 3 \\
\hline
\end{tabular}

\section{Results}

This section presents the metrics that were evaluated. Although HMD* and $\tau_{m o d} *$ are not completely independent (because the definition of $\tau_{m o d} *$ is dependent on HMD*), this study may be able to provide some insight into the effect of HMD* and $\tau_{\text {mod }} *$ on the metrics. Because DWC1 and DWC3 have the same $\tau_{\text {mod }} *$ but different HMD*s, HMD* likely causes any difference in metrics between DWC1 and DWC3. Likewise, because DWC1 and DWC2 have similar HMD* but different $\tau_{\text {mod }} *, \tau_{\text {mod }} *$ likely causes any difference in metrics between DWC1 and DWC2. Results for low C-SWaP UAS will be presented first (Section A), and results for Phase 1 UAS will be presented in the following subsection (Section B). All results are for mitigated encounters, unless otherwise specified.

\section{A. Low C-SWaP UAS Results}

\section{A.1 Safety Metrics}

Fig. 3 shows the NMAC risk ratios (left) and LoDWC ratios (right) for the four DWC candidates. The causes of NMACs after DAA maneuvers include the following:

1. Intruder and ownship maneuvers

2. Surveillance volume limitation and sensor uncertainties (none in this simulation)

3. Guidance ineffectiveness or instability of guidance

4. Pilot response unable to keep up with the situation

For any specific encounter leading to an NMAC, all causes could have contributed to it. For example, analysis of a few select encounters leading to NMACs indicates an intruder maneuver near the UAS, causing the conflict guidance bands to saturate, leaving no conflict-free heading available. In this situation, the WCR guidance comes up, is executed, but changes turn directions multiple times during the UAS's maneuver. Combined with the pilot response delay, this instability of guidance can cause a chase situation, resulting in the DAA system's failure to avoid an NMAC. The LoDWCs result from similar causes.

Fig. 5 puts NMACs into two categories. Unresolved NMAC risk is comprised of encounters that lead to nominal NMACs (i.e., without a DAA system) and which still have NMACs with the DAA system. Induced NMAC is comprised of encounters that do not have nominal NMACs but develop into NMACs with the DAA maneuver in response to DAA guidance. The unresolved and induced LoDWCs are defined in a similar way.

An important observation of the NMAC risk ratios shown in Fig. 3 is that they are all fairly small and there is no statistically significant difference among them, even when compared to the Phase 1 DWC, This suggests that, given sufficient surveillance volume (infinite for this simulation) and small surveillance uncertainties (none for this simulation), all candidate DWCs are likely to be acceptable in terms of their resulting DAA performance to avoid NMACs. Interestingly, the Phase 1 DWC does not perform better with its large volume. In reality, finite surveillance volume and sensor uncertainties will increase the NMAC risk ratios.

In terms of LoDWC ratio, DAA is unable to avoid LoDWC in about $10 \%$ of the encounters. DWC2 has the lowest value of .09 but is only marginally lower than DWC1's .10. DWC3, DWC4, and the Phase 1 DWC all have comparable values (0.12). Unresolved risk ratios comprise the majority of the LoDWC counts. Intruder and ownship maneuvers 
are likely to be the main cause of these unresolved LoDWCs. Adding a buffer to the heading selected by the pilot response model was tested in this study, but showed no improvement.

NMAC Risk Ratios

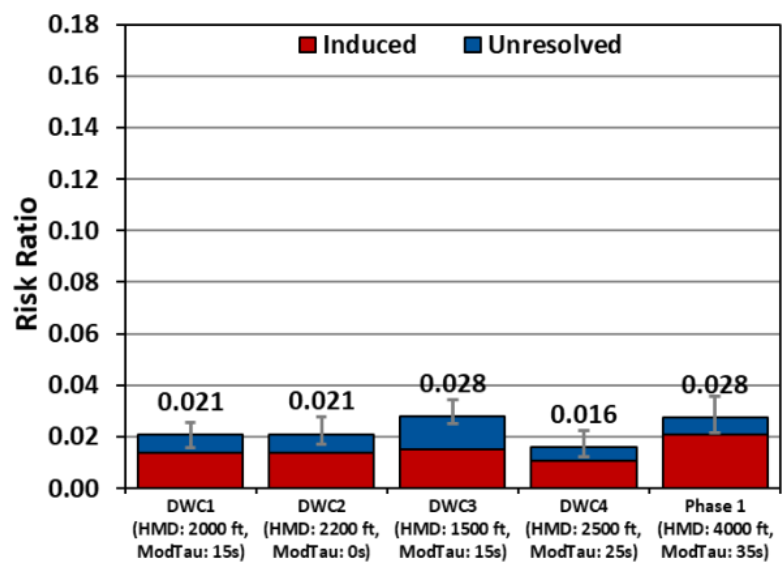

Loss of Well Clear Ratios

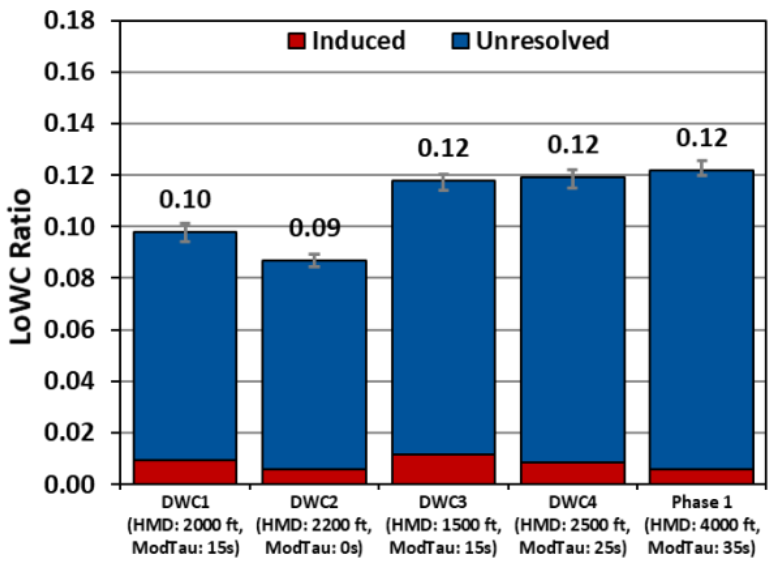

Fig. 3 Safety Ratios

One of the trends observed among the encounters with NMACs was that the intruder or ownship had a nominal (scheduled) maneuver late during the encounter. Nominal maneuvers are maneuvers that are part of the original unmitigated encounter. To analyze the impact of this trend, NMAC risk ratios and LoDWC ratios were computed for the subset of encounters where neither the ownship nor intruder has a nominal maneuver within 30 seconds of nominal TCA (Fig. 4), and for the subset of encounters with late maneuvers-i.e., where either the ownship or intruder has a nominal maneuver within 30 seconds of nominal TCA (Fig. 5). Compared to the safety ratios for all encounters (Fig. 3), the safety ratios without maneuvering (Fig. 4) are much lower. Phase 1 now has the highest LoDWC ratio, whereas previously, the LoDWC ratios for all encounters were comparable among DWC3, DWC4, and Phase 1. Since the Phase $1 \mathrm{DWC}$ is the largest and has the longest timeline, it is likely that maneuvers that occurred 30 seconds or more before the TCA contribute a sizable number to the LoDWC risk.

NMAC Risk Ratios

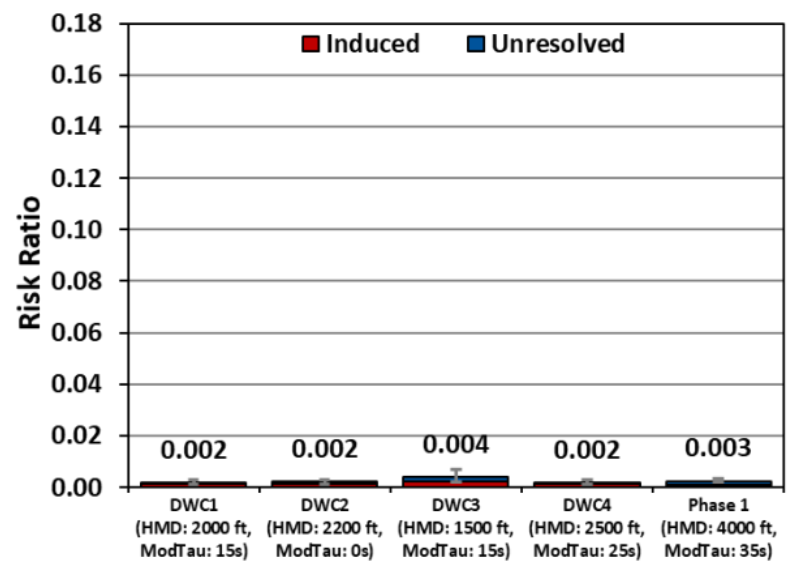

Loss of Well Clear Ratios

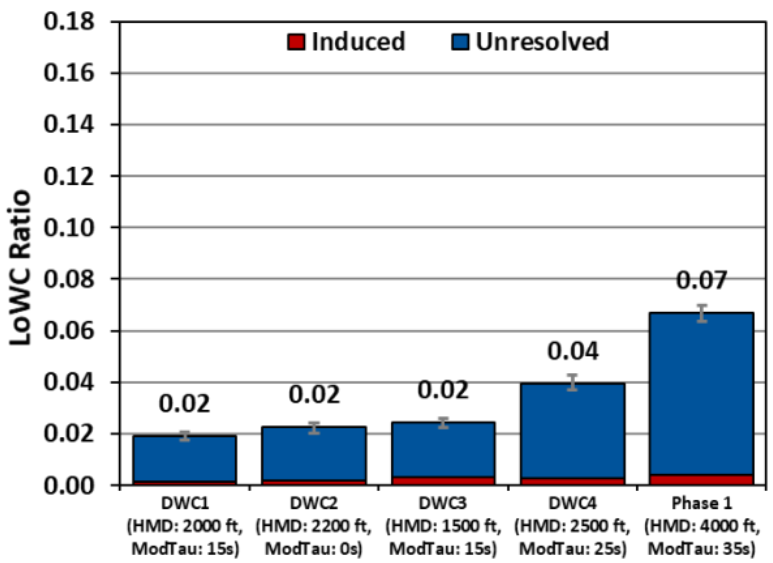

Fig. 4 Safety Ratios without Ownship or Intruder Maneuvering

In contrast, the NMAC risk ratios and LoDWC ratios with late maneuvers (Fig. 5) are much higher compared to ratios where encounters with maneuvers are excluded. This suggests that one reason the risk ratios for all encounters are comparable is because the risk comes primarily from encounters with late maneuvers (and hence, late alerts), which cannot be mitigated by any DWC. DWC3 is the least robust to late maneuvers with the highest LoDWC ratio, the second-highest NMAC risk ratio, and the most unresolved NMACs. 
NMAC Risk Ratios



Loss of Well Clear Ratios

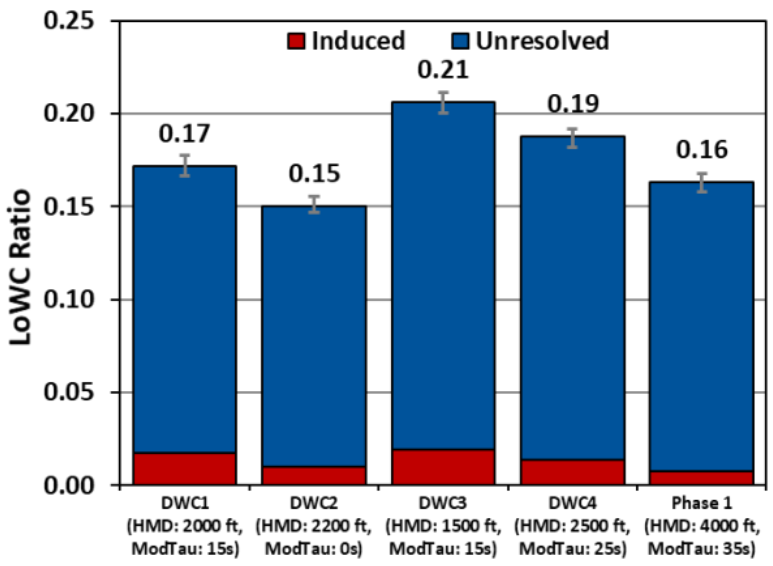

Fig. 5 Safety Ratios with Ownship or Intruder Late Maneuvering

The system operating characteristic (Fig. 6) allows simultaneous evaluation of safety and operational suitability. The alert ratio measures the alert frequency relative to the unmitigated NMAC frequency, so it is independent of the encounter definition. Ideally, low values of both metrics are preferred and therefore the closer a system is to the origin the better. HMD* appears to have the largest effect on alert ratio; DWC1 and DWC3 have the same $\tau_{\text {mod }}{ }^{*}$, but DWC1 has a larger HMD* and alerts more frequently. DWC3 has the lowest alert ratio because it has the smallest HMD*.



Fig. 6 System Operating Characteristic for Low C-SWaP Encounters

\section{A.2. Operational Suitability Metrics}

Alerting time and range are computed based on the first alert of any level that occurs in an encounter. Alerting time is the projected time to unmitigated LoDWC when the alert occurs. Only encounters that have an unmitigated LoDWC are included in this metric. Fig. 7 shows the cumulative distribution function for alerting time and range. The cumulative distribution function is the probability that alerting time or range will be less than or equal to the values on the x-axis. For example, the alerting range plot shows that $60 \%$ of encounters run with DWC4 alert at range of 3 NM or less, and all encounters run with DWC4 alert within 6 NM.

Mitigated encounters that, with DAA maneuvers, still result in a LoDWC (dashed lines) have on average later alert times and shorter ranges than all encounters with an alert (solid lines). This suggests that many LoDWCs may be caused by late nominal (non-DAA) maneuvers. Alerting time and range are driven more by $\tau_{\text {mod }}$ than by HMD (as indicated by the larger difference between DWC1 and DWC2 than between DWC1 and DWC3). DWC2, which has no $\tau_{\text {mod }}$, has the earliest alerting time relative to LoDWC and the smallest alerting range. This implies the surveillance range required to provide the alerting timeline for DWC2 is likely smaller than those for other DWCs. 



Fig. 7 Alerting Time and Range. Solid lines are all encounters. Dashed lines are encounters with LoDWC.

\section{A.3. Effect of Surveillance Range on Safety Metrics}

To assess the potential impact of limited surveillance ranges on safety, NMAC risk ratios and LoDWC ratios were compared for simulations run with a $2 \mathrm{NM}, 3 \mathrm{NM}$, and $4 \mathrm{NM}$ surveillance range limit (shown in Fig. 8). The NMAC risk ratios for DWC 1,2, and 3 are largely insensitive to reduced surveillance ranges. On the other hand, the NMAC risk ratios for DWC4 and Phase 1 experience large increases when the surveillance range is reduced to $2 \mathrm{NM}$. For the DWC4 and Phase 1 volumes, the intruder is sometimes not observed until loss of Well Clear has already occurred (particularly during higher speed encounters), and DAIDALUS's regain DWC guidance is likely not as effective in avoiding NMACs as its maintain DWC guidance. The LoDWC risk ratios for DWC1 and DWC2 increase noticeably while the value for DWC3 stays constant at 2 NM. The LoDWC risk ratios for DWC4 and the Phase DWC increase the most because $2 \mathrm{NM}$ is inside of their DWC volume for some encounters.


Fig. 8 Safety Ratios for Limited Surveillance Ranges

\section{B. Phase I UAS Results}

Analysis of the Phase 1 UAS encounters was performed on a set of one million encounters between one Phase 1 UAS and one non-cooperative intruder. The same DWC volumes used to evaluate the low C-SWaP UAS encounters were used to evaluate the Phase 1 UAS encounters in order to understand the effect of high speed UAS and baseline any additional differences when comparing to Phase 1 results. For the Phase 1 UAS results, truth surveillance data were constrained by the Phase 1 radar field of view, defined as $\pm 8 \mathrm{NM}$ range, $\pm 15^{\circ}$ elevation, and $\pm 110^{\circ}$ azimuth. 


\section{B.1. Safety Metrics}

Fig. 9 shows the NMAC risk ratios (left) and LoDWC ratios (right). Results similar to those for low C-SWaP UAS are desirable because this would corroborate the notion that the same DWC can be applied to both low C-SWaP UAS and Phase 1 UAS. As with the low C-SWaP UAS results, the NMAC risk ratios and LoDWC ratios are comparable among the DWC candidates. However, the risk ratios are approximately five times larger than the risk ratios for low C-SWaP UAS, and the LoDWC ratios are approximately two times larger than the LoDWC ratios for low C-SWaP UAS. This difference is primarily due to the limited $110^{\circ}$ bearing range, which results in undetected intruders and therefore unresolved NMACs and LoDWCs. When the Phase 1 risk ratios and LoDWC ratios are computed without bearing and elevation limitations (as shown in Fig. 10), the results are much closer to the ratios obtained using the low C-SWaP encounter set (Fig. 3).
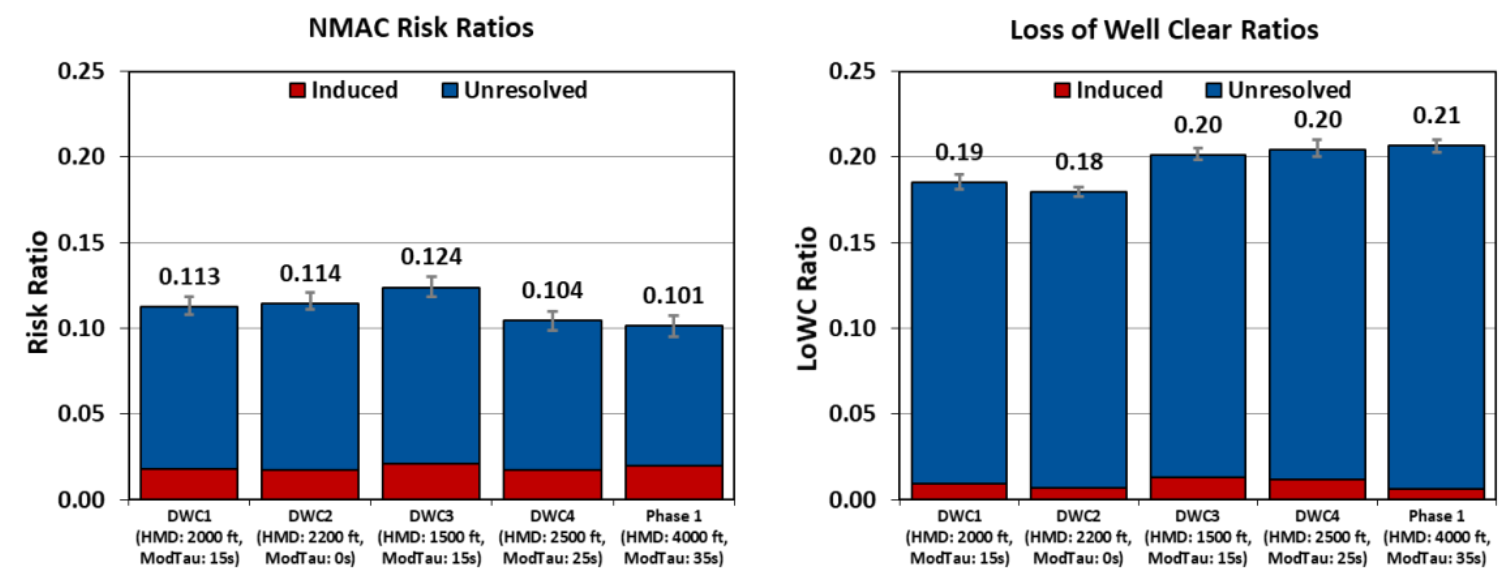

Fig. 9 Safety Ratios
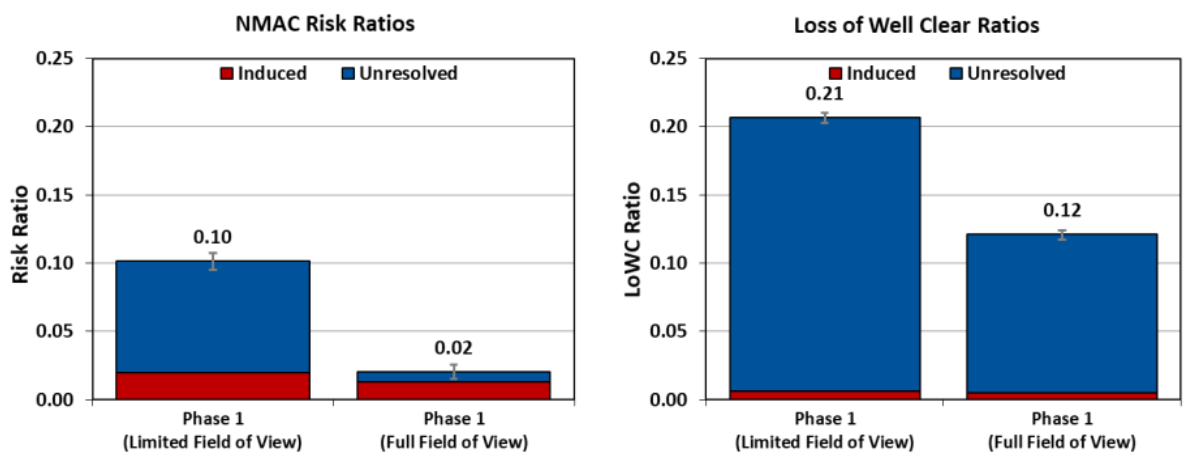

Fig. $10 \quad$ Safety Ratios with Full Field of View

Fig. 11 compares the above safety metrics to the alert ratio, providing insight into the potential tradeoff between safety and operational suitability. Like the low C-SWaP encounter set (Fig. 6), HMD has the largest effect on alert ratio; larger volumes result in significantly higher alert ratios, while maintaining similar safety. 


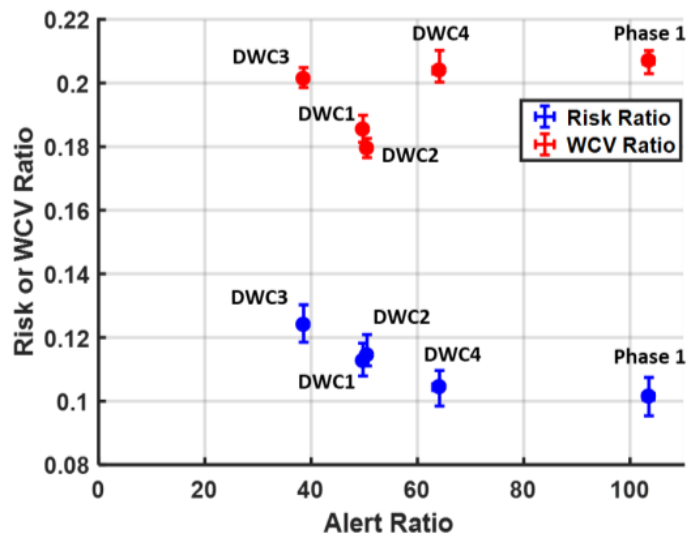

Fig. 11 System Operating Characteristic for Phase 1 Encounters

\section{B.2 Operational Suitability Metrics}

Fig. 12 illustrates the time of alert, prior to unmitigated LoDWC (left) and range at time of first alert (right). The time of alerts has a noticeable negative portion (the non-zero cumulative frequency at 0 alert time) because many intruders enter the DWC volume undetected by the limited surveillance volume. As expected, the larger Phase 1 volume alerts sooner and at larger separations. Similar trends are seen compared to the low C-SWaP UAS analysis (Fig. 7); again, alerting time and range are driven more by $\tau_{\text {mod }}$ than HMD. DWC2, which has no $\tau_{m o d}$, has the earliest alerting time relative to LoDWC and the smallest alerting range.
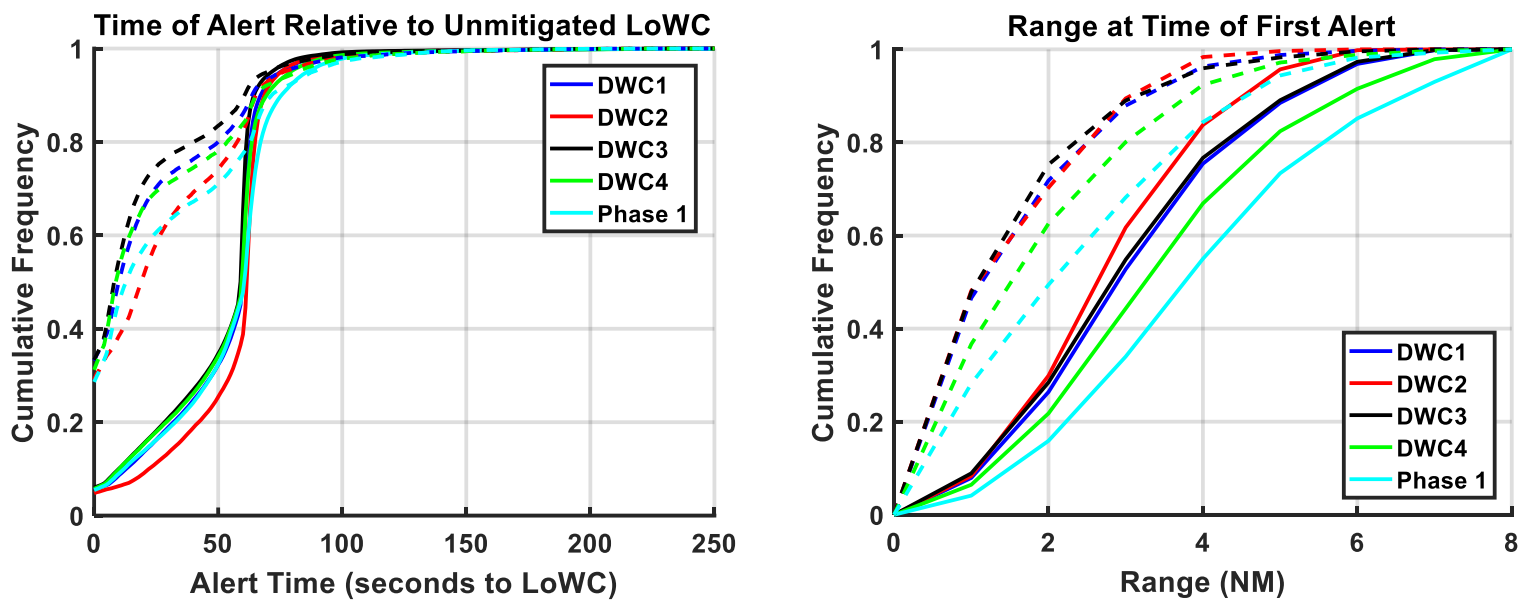

Fig. 12 Alerting Time and Range. Solid lines are all encounters. Dashed lines are encounters with LoDWC.

\section{B.3 Effect of Ownship Speed on Safety}

The Phase 1 UAS encounters encompass both low C-SWaP and high-performance aircraft against a VFR intruder. To assess the sensitivity of the safety metrics to ownship speed, the results were binned by maximum ownship speed shown in Table 5. The relative frequency of encounters in each of these bins is shown in Fig. 13.

Table 5 - Ownship Speed Bins

\begin{tabular}{|l|l|}
\hline Bin & Maximum Ownship Speed Range \\
\hline 1 & $40-100$ knots \\
\hline 2 & $100-150$ knots \\
\hline 3 & $150-200$ knots \\
\hline 4 & $200+$ knots \\
\hline
\end{tabular}






Fig. 13 Frequency of Encounters per Bin

Fig. 14 shows the risk ratios binned by maximum ownship speed. There are no mitigated NMACs when the ownship aircraft has a maximum speed greater than 200 knots (Speed Bin 4), but this could be caused by the few number of encounters in Speed Bin 4. Likewise, DWC3 appears to induce NMACs for Speed Bin 3, but this is not statistically significant. In general, the risk ratios in Speed Bin 1 are highest, mainly because UAS in Speed Bin 1 are more likely to have overtaking aircraft from the rear, outside the radar's field of view. UAS in speed bins 2,3 , and 4 usually fly faster than the intruder and are therefore less likely to have undetected intruders approach from the rear.
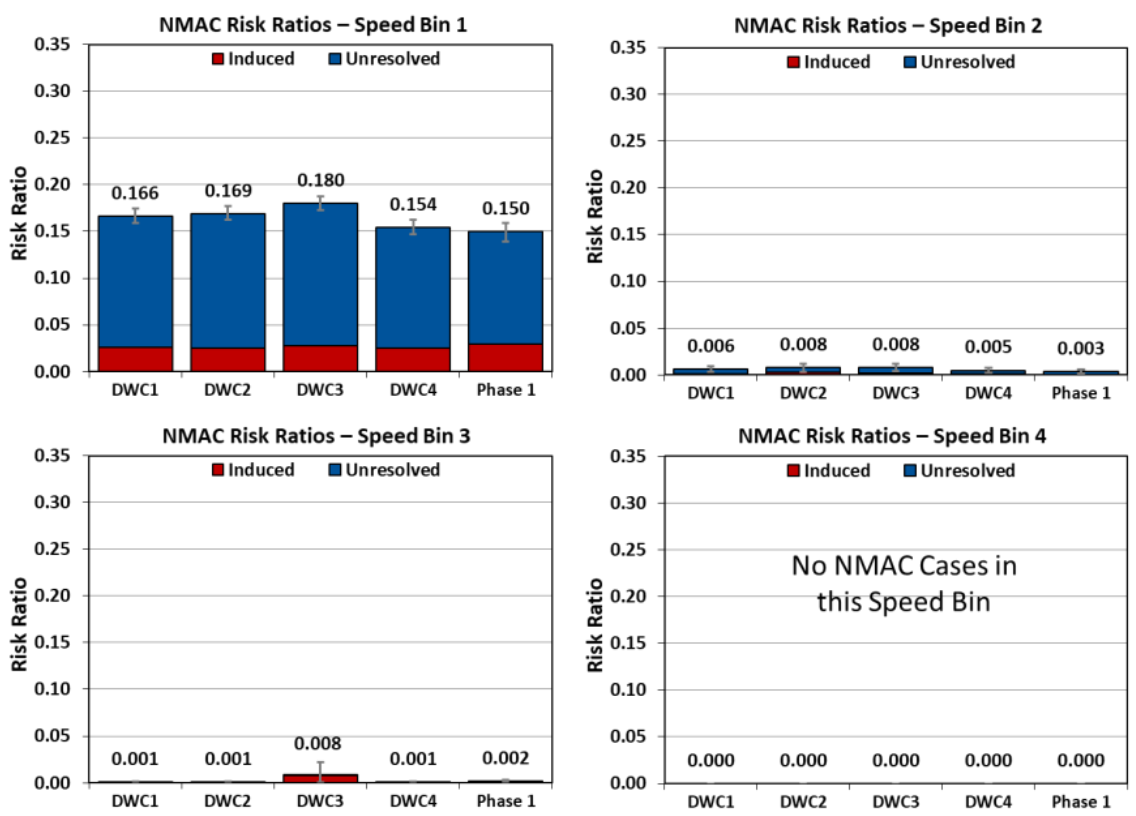

Fig. 14 NMAC Risk Ratios Binned by Speed

Fig. 15 shows the LoDWC ratios binned by maximum ownship speed. For slower aircraft (Speed Bin 1), LoDWC ratios are all comparable. For faster aircraft (Speed Bins $2,3,4), \tau_{\text {mod }} *$ seems to have a larger effect (low $\tau_{\text {mod }} *$ leads to lower LoDWC ratios). DWC2, the only DWC with a zero $\tau_{\text {mod }}{ }^{*}$, consistently leads to the lowest LoDWC risk ratio.

Although not modeled in this work, sensor uncertainties are likely to increase the NMAC and LoDWC risk ratio. For example, a MITRE study [9] yielded a NMAC risk ratio of 0.22 for a class of UAS similar to those in the Speed Bin 1 when taking into account sensor uncertainties (compared to 0.15 without uncertainty in this simulation), and a LoDWC risk ratio of 0.42 for a class of UAS similar to those in the Speed Bin 1 when taking into account sensor uncertainties (compared to 0.28 without uncertainty in this simulation). 

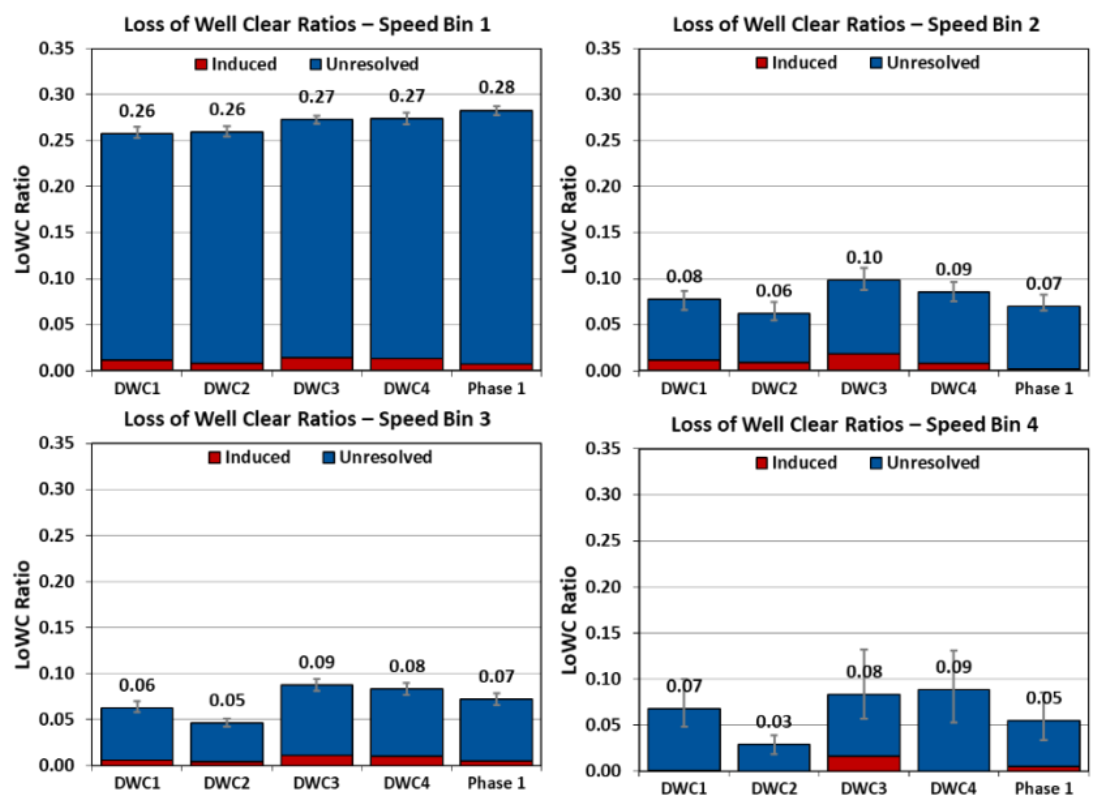

Fig. 15 LoDWC Ratios Binned by Speed

\section{Conclusion and Future Work}

This analysis evaluated four potential Detect-and-Avoid (DAA) Well Clear (DWC) definitions for UAS encountering non-cooperative aircraft using safety and operational suitability metrics. Two sets of encounters were used to evaluate the metrics in the two operational contexts of interest: one with encounters between low C-SWaP UAS and non-cooperative intruders, and another with encounters between Phase 1 UAS and non-cooperative intruders.

The low C-SWaP UAS analysis shows that NMAC risk and LoDWC ratios are not sensitive to DWC parameters under perfect surveillance. Furthermore, safety and operational suitability are not dependent on $\tau_{m o d}{ }^{*}$; this indicates that $\tau_{m o d} *$ may not be necessary in a DWC definition. $\tau_{m o d} *$ affects mainly alerting performance (timing and range), whereas safety and operational suitability are of primary concern for a DAA Well Clear definition for low C-SWaP UAS. Because $\tau_{\text {mod }}$ requires a larger tracking range without providing any additional safety or operational suitability benefit, the results give preference to DWC2, which has no temporal parameter.

The Phase 1 UAS analysis results generally follow the same trends as the low C-SWaP UAS analysis results. In terms of safety, with no radar field of view applied, the risk ratio and LoDWC ratios for Phase 1 UAS are comparable to those for low C-SWaP UAS. This seems to support the hypothesis that the final non-cooperative DWC definition for low C-SWaP UAS is also applicable to Phase 1 UAS. With the Phase 1 radar field of view applied, NMAC and LoDWC risk ratios increase noticeably due to a large number of undetected intruders approaching from the rear of the UAS.

The Phase 1 UAS analysis results were also binned by maximum ownship speed. As ownship speed increases, the LoDWC risk ratios seem to be driven more by $\tau_{m o d} *$ than by HMD*. A smaller $\tau_{m o d} *$ seems to have little effect on safety at low speeds and also reduces the safety ratios at high speeds. This again corroborates the result that $\tau_{\text {mod }}$ is not needed in a definition for low C-SWaP UAS and Phase 1 UAS against non-cooperative intruders.

Based on the findings presented in this paper as well as a companion paper [15], SC-228 has selected DWC2 (2200 $\mathrm{ft}, 450 \mathrm{ft}, 0 \tau_{\text {mod }}{ }^{*}$ ) for low C-SWaP UAS and Phase 1 UAS encountering non-cooperative aircraft.

The following tasks can be considered as follow-on efforts to this analysis:

- Additional safety analyses using DWC2, and potentially test lower HMD values with $0 \tau_{\text {mod }}$ to see if the DAA Well Clear definition can be further reduced

- Access the effects of intruder speed or relative speed on safety performance

- Development and validation of low C-SWaP sensor requirements, and associated guidance and alerting

- Human factors evaluation of low C-SWaP DWC to validate the alerting timeline, and understand ATC response to and pilot acceptability of the low C-SWaP DWC. 


\section{References}

[1] S. P. Cook, D. Brooks, R. Cole, D. Hackenberg and V. Raska, "Defining Well Clear for Unmanned Aircraft Systems," AIAA Infotech@ Aerospace, Kissimmee, FL, 2015.

[2] D. Walker, "FAA Position on Building Consensus Around the SARP Well-Clear Definition," in RTCA Special Committee 228, 2014.

[3] Minimum Operational Performance Standards (MOPS) for Detect and Avoid (DAA) Systems, DO-365, RTCA. Inc., 2017.

[4] Minimum Operational Performance Standards (MOPS) for Air-to-Air Radar for Traffic Surveillance, DO-366, RTCA. Inc., 2017.

[5] M. G. Wu, A. C. Cone, S. Lee, C. Chen, M. W. M. Edwards, Jack and D. P., "Well Clear Trade Study for Unmanned Aircraft System Detect and Avoid with Non-Cooperative Aircraft," AIAA Aviation Conference, Atlanta, GA, 2018.

[6] S. George, G. Satapathy, G. Manikonda, M. Refai and R. Dupee, "Build 8 of the Airspace Concept Evaluation System," in AIAA Modeling and Simulation Technologies Conference, 2011.

[7] A. Weinert, E. Harkleroad, J. Griffith, M. Edwards and M. Kochenderfer, "Uncorrelated Encounter Model of the National Airspace System Version 2.0 (ATC-404)," Lincoln Laboratory, Lexington, MA, 2013.

[8] C. Muñoz, A. Narkawicz, G. Hagen, J. Upchurch, A. Dutle, M. Consiglio and J. Chamberlain, "DAIDALUS: Detect and Avoid Alerting Logic for Unmanned Systems," in 34th IEEE/AIAA Digital Avionics Systems Conference, Prague, Czech Republic, 2015.

[9] M. Hall, J. Kaznocha, T. Lester, S. Sherman and N. Reep, "SC-228 DAA Phase 1 MOPS Verification \& Validation Simulation," MITRE Corporation, Bedford, MA, 2017.

[10] Minimum Operational Performance Standards for Traffic Alert and Collision Avoidance System II (TCAS II) Version 7.1, Washington, DC: DO-185B, RTCA, 2008.

[11] S. Ayyalasomayajula, R. Sharma, W. F. A. Trani, N. Hinze and S. Spencer, "UAS Demand Generation Using Subject Matter Expert Interviews and Socio-Economic Analysis," in Proceedings of the AIAA Aviation Conference, 2015.

[12] A. Nuic, D. Poles and V. Mouillet, "An Advanced Aircraft Performance Model for Present and Future ATM Systems," International Journal of Adaptive Control and Signal Processing, Vol. 24, No. 10, pp. 850-866, 2010.

[13] R. Guendel, M. Kuffner and D. Maki, "A Model of Unmanned Aircraft Pilot Detect and Avoid Maneuver Decisions," Massachusetts Institute of Technology Lincoln Laboratory Project Report ATC-434, 2017.

[14] B. Efron, "Better Bootstrap Confidence Intervals," Journal of the Americal Statistical Association, vol. 82, no. 397, pp. 171-185, 1987.

[15] A. C. Cone, M. G. Wu and S. Lee, "Detect-and-Avoid Alerting Performance for High-Speed UAS and NonCooperative Aircraft," in AIAA Aviation Conference, 2019. 